\title{
Broadband Spectroscopy of Thermodynamic Magnetization Fluctuations through a Ferromagnetic Spin-Reorientation Transition
}

\author{
A. L. Balk, ${ }^{1}$ F. Li, ${ }^{2,3,{ }^{*}}$ I. Gilbert, ${ }^{4}$ J. Unguris, ${ }^{4}$ N. A. Sinitsyn, ${ }^{2}$ and S. A. Crooker ${ }^{1}$ \\ ${ }^{1}$ National High Magnetic Field Laboratory, Los Alamos National Laboratory, \\ Los Alamos, New Mexico 87545, USA \\ ${ }^{2}$ Theoretical Division, Los Alamos National Laboratory, Los Alamos, New Mexico 87545, USA \\ ${ }^{3}$ Center for Nonlinear Studies, Los Alamos National Laboratory, Los Alamos, New Mexico 87545, USA \\ ${ }^{4}$ Center for Nanoscale Science and Technology, National Institute of Standards and Technology, \\ Gaithersburg, Maryland 20899, USA
}

(Received 26 January 2018; published 21 September 2018)

\begin{abstract}
We use scanning optical magnetometry to study the broadband frequency spectra of spontaneous magnetization fluctuations, or "magnetization noise," in an archetypal ferromagnetic film that can be smoothly tuned through a spin-reorientation transition (SRT). The SRT is achieved by laterally varying the magnetic anisotropy across an ultrathin $\mathrm{Pt} / \mathrm{Co} / \mathrm{Pt}$ trilayer, from the perpendicular to in-plane direction, via graded $\mathrm{Ar}^{+}$irradiation. In regions exhibiting perpendicular anisotropy, the power spectrum of the magnetization noise $S(\nu)$ exhibits a remarkably robust $\nu^{-3 / 2}$ power law over frequencies $\nu$ from $1 \mathrm{kHz}$ to $1 \mathrm{MHz}$. As the SRT region is traversed, however, $S(\nu)$ spectra develop a steadily increasing critical frequency $\nu_{0}$, below which the noise power is spectrally flat, indicating an evolving low-frequency cutoff for magnetization fluctuations. The magnetization noise depends strongly on applied in- and out-of-plane magnetic fields, revealing local anisotropies and also a field-induced emergence of fluctuations in otherwise stable ferromagnetic films. Finally, we demonstrate that higher-order correlators can be computed from the noise. These results highlight broadband spectroscopy of thermodynamic fluctuations as a powerful tool to characterize the interplay between thermal and magnetic energy scales, and as a means of characterizing phase transitions in ferromagnets.
\end{abstract}

DOI: $10.1103 /$ PhysRevX.8.031078

Subject Areas: Condensed Matter Physics, Magnetism, Nanophysics

\section{INTRODUCTION}

Spontaneous magnetization fluctuations can occur in ferromagnetic materials, even in thermal equilibrium, particularly when the magnetic anisotropy energy becomes comparable to or less than the available thermal energy [1-7]. These intrinsic thermodynamic fluctuations encode valuable information about the magnetization dynamics of the system itself, because their frequency spectrum $S(\nu)$ is intimately and necessarily related to the dissipative (imaginary) part of the magnetic susceptibility $\chi^{\prime \prime}(\nu)$, in accord with the fluctuation-dissipation theorem [2,8] [namely, $\chi^{\prime \prime}(\nu) \sim\left[\nu /\left(k_{B} T\right)\right] S(\nu)$, where $k_{B} T$ is the thermal energy]. Spectroscopy of this intrinsic "magnetization noise" can therefore provide an alternative and entirely passive means

\footnotetext{
*Present address: School of Physics and Electronics, Hunan University, Changsha 410082, China.

Published by the American Physical Society under the terms of the Creative Commons Attribution 4.0 International license. Further distribution of this work must maintain attribution to the author(s) and the published article's title, journal citation, and DOI.
}

of measuring magnetization dynamics that does not require driving, exciting, or perturbing the system away from thermal equilibrium-in contrast with most conventional methods for measuring magnetic resonance or ac susceptibility.

A fascinating and technologically relevant test bed in which to study thermodynamic magnetization fluctuations is thin ferromagnetic films, because magnetic anisotropy energies can be readily engineered over a very wide range via material choice and by growth and postprocessing conditions $[9,10]$. This tunability arises from the competition and delicate balance between shape anisotropy (which generally favors in-plane magnetic alignment) and interfacial anisotropy (which can favor out-of-plane magnetic alignment for certain material combinations). Especially interesting are films in which the total magnetic anisotropy is continuously tuned to and through zero, by laterally varying either the film's thickness $[11,12]$ or its degree of interfacial disorder $[13,14]$. In this case, the film can exhibit a spin-reorientation transition (SRT) [15-18] wherein the direction of ferromagnetic ordering transitions from out of plane (perpendicular) to in plane. In perpendicularly magnetized films with small net anisotropy, it is well established that the combination of exchange and dipolar 
energies leads to the formation of mazelike patterns of magnetic domains $[19,20]$, as observed and studied extensively in ultrathin films of Fe/Ni [21-24], Co/Pt [25-27], and $\mathrm{CoFeB}$ [28]. As the SRT is approached and the net magnetic anisotropy is reduced to zero, it has recently been observed that these domain patterns begin to fluctuate markedly in time. These thermodynamic magnetization fluctuations have been measured by electron microscopy [23,29-31], magneto-optic Kerr effect (MOKE) [26,32], $\mathrm{x}$-ray scattering [33], and transport [34], revealing spatial correlations, topological effects, suceptibilities, and higherorder anisotropy. However, timescales of these measurements are typically slow, being limited to $>1 \mathrm{~ms}$. Fluctuations at frequency scales faster than $1 \mathrm{kHz}$ were not resolved. However, the full frequency spectrum of these fluctuations contains rich information about the distributions of relaxation rates and the evolution of the anisotropy landscape through the SRT, which provide important insight for developing theoretical models.

To address this need and to complement these recent studies, here we develop a fast optical magnetometer to investigate the broadband frequency spectrum of thermodynamic magnetization fluctuations in ferromagnetic films that are smoothly tuned through a SRT. Using $\mathrm{Pt} / \mathrm{Co} / \mathrm{Pt}$ trilayers with laterally graded magnetic anisotropy, we find that the frequency spectrum of the magnetization noise $S(\nu)$ exhibits a remarkably robust $\nu^{-3 / 2}$ power law from $1 \mathrm{kHz}$ to $1 \mathrm{MHz}$

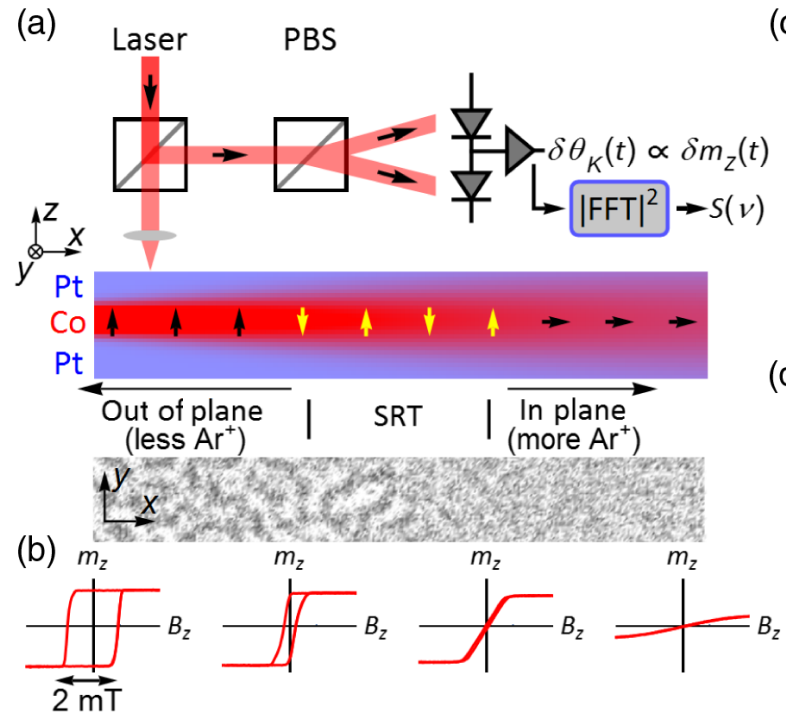

in regions exhibiting perpendicular magnetization, indicating a broad distribution of fluctuation rates. However, as the SRT region is traversed, $S(\nu)$ develops a steadily increasing critical frequency $\left(\nu_{0}\right)$ below which the noise tends towards spectrally flat, indicating a minimum relaxation rate that becomes increasingly fast through the SRT. The magnetization fluctuations also depend strongly on applied magnetic fields, which can be understood within the context of the fluctuationdissipation theorem and from considerations of the magnetic free energy. Field-dependent maps of the noise reveal detailed local magnetic anisotropies, and field-induced fluctuations are found to emerge even in trilayers with nominally stable perpendicular ferromagnetism. Finally, we show that these methods can be used to analyze higher-order noise correlations, which can probe non-Gaussian noise and time-reversal breaking effects. These results demonstrate that broadband optical detection of magnetization fluctuations provides a powerful and straightforward method for studying the subtle interplay between thermal energy and magnetic anisotropy that can drive phase transitions in ferromagnets, and in particular the spin-reorientation transition in ultrathin films.

\section{Pt/Co/Pt TRILAYERS WITH LATERALLY GRADED MAGNETIC ANISOTROPY}

Figure 1(a) depicts the experiment and samples. The samples are $\mathrm{Ta}(3.8 \mathrm{~nm}) / \operatorname{Pt}(3.9 \mathrm{~nm}) / \mathrm{Co}(0.8 \mathrm{~nm}) /$

(c)
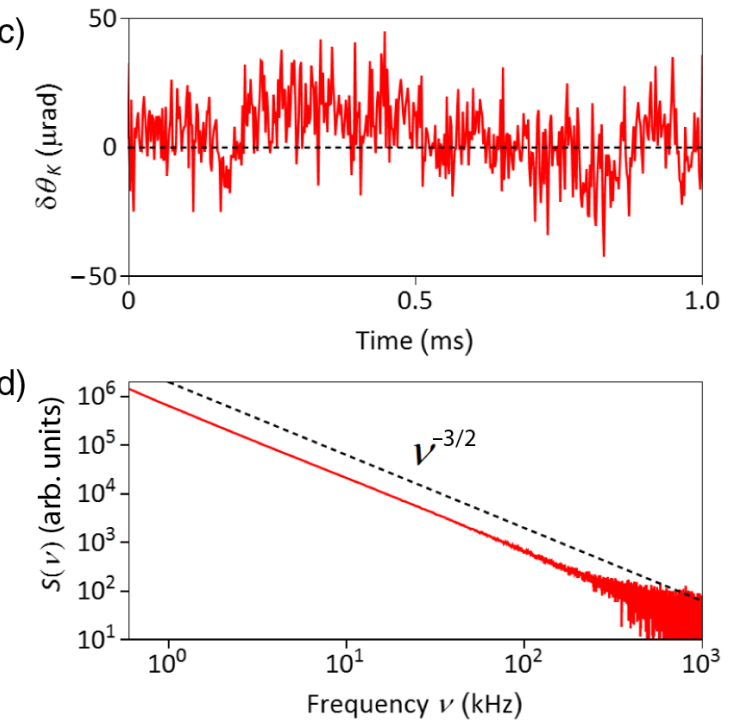

FIG. 1. (a) Experimental schematic. A scanning broadband optical magnetometer based on polar MOKE measures the spontaneously fluctuating out-of-plane magnetization $\delta m_{z}(t)$ within a small $(4 \mu \mathrm{m})$ spot on the sample. The power spectral density of this magnetization noise $S(\nu)$ is continuously computed and averaged in real time (PBS: polarizing beamsplitter, FFT: fast Fourier transform). The sample is a $\mathrm{Pt} / \mathrm{Co} / \mathrm{Pt}$ trilayer irradiated with a laterally varying dose of $\mathrm{Ar}^{+}$, generating a lateral gradient of interfacial magnetic anisotropy. Red and blue colors depict $\mathrm{Co}$ and Pt layers; arrows depict the net magnetization direction, with yellow arrows indicating the region where the spin reorientation transition (SRT) occurs. (b) The magnetic contrast image of the trilayer surface (adapted from Ref. [32]) shows the mazelike domain patterns that form in graded trilayers of this type. The patterns become blurry near the SRT as fluctuations increase and occur faster than the image acquisition time. Conventional MOKE magnetization measurements $m_{z}\left(B_{z}\right)$ show that the coercive field $\mu_{0} H_{c} \rightarrow 0$ as the SRT is approached. (c) Characteristic Kerr rotation fluctuations $\delta \theta_{K}(t)$ reveal the thermodynamic magnetization fluctuations $\delta m_{z}(t)$. (d) $S(\nu)$ falls as a $\nu^{-3 / 2}$ power law over the measured $1 \mathrm{kHz}-1 \mathrm{MHz}$ range in regions of the trilayer exhibiting perpendicular magnetization. Discussion of measurement uncertainties in this and subsequent figures can be found in the Supplemental Material [35]. 
$\operatorname{Pt}(1.9 \mathrm{~nm})$ ultrathin ferromagnetic films sputtered on $\mathrm{Si}$ substrates under high vacuum conditions. Conventional polar MOKE measurements of the average out-of-plane magnetization $\left(m_{z}\right.$ versus $\left.B_{z}\right)$ confirm that the as-grown films have perpendicularly oriented magnetization, with approximately square hysteresis loops and coercive fields $\mu_{0} H_{c} \approx 2 \mathrm{mT}$. To tune the magnitude and direction of the net magnetic anisotropy, we irradiate the samples with a laterally varying dose of low-energy $(100 \mathrm{eV}) \mathrm{Ar}^{+}$, which generates disorder and reduces the interfacial (perpendicular) magnetic anisotropy [13]. The net magnetization direction that results from the competition between interfacial and shape anisotropy is therefore oriented out of plane $(\| \pm \hat{z})$ on regions of the sample with lower disorder, but switches to in-plane orientation $(\perp \hat{z})$ on sample regions with higher disorder, as depicted. Between these regions the spinreorientation transition occurs.

Shown below the illustration of the trilayer is a magnetic contrast image of the surface of a laterally graded $\mathrm{Pt} / \mathrm{Co} / \mathrm{Pt}$ trilayer (obtained via MOKE), showing the mazelike magnetic domain patterns that exist at $B=0$ in its fully relaxed and demagnetized state. Dark and bright domains correspond to out-of-plane magnetization oriented along $+\hat{z}$ and $-\hat{z}$, respectively. As also observed in many prior studies of ultrathin ferromagnets [21,23,30,32], the characteristic size of the domains decreases as the SRT region is approached and the perpendicular magnetic anisotropy is reduced. Near the SRT, the images become increasingly blurry as thermodynamic fluctuations of the domain walls increase and become faster than the image acquisition rate (typically video rate, or $\approx 30 \mathrm{~Hz}[26,30,32]$, but as fast as $1 \mathrm{kHz}$ in recent work by Kronseder et al. [31]). Our goal is to measure the relevant timescales and frequency distributions of these magnetization fluctuations over a much broader range of timescales and deep into (and through) the SRT, where the temporal resolution of imaging techniques can be limited.

Figure 1(b) shows conventional polar MOKE measurements of $m_{z}\left(B_{z}\right)$ across the sample, confirming the graded magnetic anisotropy: The open hysteresis loops become narrower as perpendicular anisotropy is reduced, and the coercive field $\mu_{0} H_{c} \rightarrow 0$ as the SRT is approached. Near the SRT, no hysteresis is observed and the low-field magnetization is effectively linear (constant susceptibility $d m_{z} / d B_{z}$ ), indicating that the trilayer is able to relax to an equilibrium maze domain pattern on a timescale faster than the (slow) sweep rate of the applied field $B_{z}$. Beyond the SRT the magnetization direction switches to in plane and $m_{z}$ is suppressed.

\section{OPTICAL SPECTROSCOPY OF MAGNETIZATION FLUCTUATIONS}

To detect and quantify the broadband magnetization fluctuations that exist in thermal equilibrium in these $\mathrm{Pt} / \mathrm{Co} / \mathrm{Pt}$ trilayers, we developed a real-time optical noise magnetometer based on polar MOKE. A linearly polarized probe laser $(632.8 \mathrm{~nm}, \approx 1 \mathrm{~mW})$ is focused to a small (4- $\mu$ m-diameter) spot on the sample, which in turn is mounted on an $x-y$ positioning stage for lateral scanning. This arrangement exclusively measures stochastic magnetization fluctuations in the out-of-plane direction $\delta m_{z}(t)$, which impart a fluctuating Kerr rotation $\delta \theta_{K}(t)$ on the reflected probe laser that is detected using balanced photodiodes. The resulting noise signal $\delta \theta_{K}(t) \propto \delta m_{z}(t)$ is continuously digitized, processed, and averaged in real time. Typically we compute and analyze $S(\nu)$, the power spectral density of the fluctuation signal. That is, $S(\nu)=$ $\left\langle a(\nu) a^{*}(\nu)\right\rangle$, where $a(\nu)$ is the Fourier transform of $\delta m_{z}(t)$ and the brackets indicate an average over repeated measurements. Equivalently, $S(\nu)$ is the real Fourier transform of the second-order (two-point) time correlator $\left\langle\delta m_{z}(0) \delta m_{z}(t)\right\rangle$. Importantly, we note that this setup also allows for measurement and analysis of higher-than-second-order noise correlators, as demonstrated later in Sec. X.

The measurement bandwidth is determined by the speed of the photodetectors and digitizers. For these $\mathrm{Pt} / \mathrm{Co} / \mathrm{Pt}$ trilayers we use low-noise detectors and amplifiers with frequency response of several megahertz. The spectral density of the measured Kerr rotation is small, typically below $1 \mu \mathrm{rad} / \sqrt{\mathrm{Hz}}$. The fundamental photon shot noise of the probe laser itself contributes $\sim 50 \mathrm{nrad} / \sqrt{\mathrm{Hz}}$ of (white) background noise; this constitutes the dominant source of nonmagnetic noise at frequencies above a few hundred hertz. This shot noise can be mitigated by signal averaging and background subtraction. At lower frequencies, acoustic noise and mechanical vibrations limit accurate recovery of small magnetization fluctuations. Unless otherwise noted, we therefore restrict detailed analysis to frequencies $\nu>1 \mathrm{kHz}$. These methods are adapted from studies of "optical spin noise spectroscopy" in simple paramagnetic atomic vapors and semiconductors, which are typically based on Faraday rotation at much higher $(\mathrm{MHz})$ frequencies [36-39].

Figure 1(c) shows an example of the spontaneous magnetization fluctuations $\delta m_{z}(t)$ that are measured in thermal equilibrium at an out-of-plane region of the $\mathrm{Pt} / \mathrm{Co} / \mathrm{Pt}$ trilayer, at room temperature $(T=295 \mathrm{~K})$ and without any applied magnetic field. The induced Kerr rotation signal $\delta \theta_{K}(t)$ fluctuates in time about zero with a typical standard deviation of several microradians. Figure 1(d) shows the corresponding power spectral density of this magnetization noise $S(\nu)$ averaged over many minutes. Strikingly, $S(\nu)$ exhibits a very robust powerlaw decay over the frequency range from $\nu=1 \mathrm{kHz}$ to $1 \mathrm{MHz}$, falling as $\nu^{-\alpha}$, where $\alpha \simeq 3 / 2$. As shown below, this power-law exponent is very robust and is independent of changes in magnetic anisotropy, temperature, and small applied magnetic fields $B$.

In general, power-law dynamics indicate that a system cannot be characterized by a single timescale but rather 
exhibits a broad distribution of relaxation and fluctuation timescales [40-42]. Given that many magnetic domains and fluctuating domain walls are simultaneously probed within the focused laser spot, it is therefore worthwhile to ask what distribution of timescales or relaxation rates might generate the measured $S(\nu) \sim \nu^{-3 / 2}$ noise spectrum.

In a simple case, consider a system comprising many independent simple fluctuators, each characterized by a single exponentially decaying correlation time $\tau_{i}$ [i.e., $\langle m(0) m(t)\rangle \sim e^{-t / \tau_{i}}$. Individually, each fluctuator therefore contributes a Lorentzian power spectral density, $S_{i}(\nu) \sim 1 /\left(\nu^{2}+\gamma_{i}^{2}\right)$, to the total measured noise spectrum; that is, $S_{i}(\nu)$ is approximately flat up to the characteristic relaxation rate $\gamma_{i}=\tau_{i}^{-1}$ before falling off as $\nu^{-2}$ at high frequencies. In this case, the total noise spectrum is the weighted sum of many Lorentzians [42], where the weight is determined by the distribution $D(\gamma)$ of fluctuators having characteristic relaxation rate $\gamma$ :

$$
S(\nu) \propto \sum_{i} \frac{1}{\nu^{2}+\gamma_{i}^{2}} \approx \int_{\gamma_{\min }}^{\gamma_{\max }} \frac{D(\gamma)}{\nu^{2}+\gamma^{2}} d \gamma .
$$

The distribution $D(\gamma)$ determines the functional form of $S(\nu)$, and the upper and lower cutoffs prevent divergences. If $D(\gamma)$ itself follows a power law $D(\gamma) \propto \gamma^{-\beta}$, then $S(\nu) \approx$ $\nu^{-(\beta+1)}$ for $\gamma_{\min } \ll \nu \ll \gamma_{\max }$. Note that if $D(\gamma)$ is truncated below some minimum relaxation rate $\gamma_{\min }$, then $S(\nu)$ will deviate from a pure power law and will tend towards spectrally flat at low frequencies below $\gamma_{\min }$, as will become relevant in the next section. Moreover, if $D(\gamma)$ is truncated above a maximum rate $\gamma_{\max }$, then $S(\nu)$ will eventually decay as $\nu^{-2}$ at high frequencies. Therefore, the $\nu^{-3 / 2}$ noise spectrum measured in Fig. 1(d) is (at least) consistent with an ensemble of simple fluctuators with distribution $D(\gamma) \propto \gamma^{-1 / 2}$. Furthermore, no signature of $\gamma_{\min }$ or $\gamma_{\max }$ is evident in the data - at this location on the sample these limits lie well below and above our measured frequency range, respectively. While clearly an oversimplificationferromagnets are, after all, correlated systems and are not composed of independent fluctuators - these arguments help to provide a basis for understanding how changes in the functional form of $S(\nu)$ can emerge.

A broad distribution of relaxation rates can be expected in ultrathin ferromagnetic films due to microscopic variations of the magnetic exchange, anisotropy, and dipolar energies. In particular, these variations can be caused by a spatially disordered magnetic energy landscape and pinning potential arising from the interface roughness, which can be comparable to film thickness. Indeed, a disordered magnetic energy forms the basis for a variety of statistical models [43-45] which have been applied to understand, e.g., the Barkhausen (magnetic switching) noise that arises in ferromagnets that are driven by a changing applied magnetic field. Predictions and measurements [46,47] of the Barkhausen noise spectrum often yield power laws with exponents in the range of -1.5 to -2 , in potential correspondence with the value of $\alpha$ determined in our studies of purely thermodynamic (undriven) magnetization fluctuations. We also note that studies of domain wall kinetics in nanowires revealed $\nu^{-3 / 2}$ power-law dynamics that were attributed to domain wall diffusion in the presence of a disordered pinning potential [48], a scenario that may also apply (with suitable dimensionality considerations) to the case of ultrathin magnetic films.

\section{LATERALLY SCANNING THROUGH THE SRT AT FIXED TEMPERATURE}

Having established a $\nu^{-3 / 2}$ power-law spectrum of magnetic fluctuations in a region of the trilayer exhibiting out-of-plane ferromagnetism, we now explore the much more interesting question of how the fluctuation spectrum evolves when the net magnetic anisotropy is reduced through zero and the sample undergoes a SRT. Figure 2 shows $S(\nu)$ as the probe laser is scanned laterally across the sample, beginning (on the left) at a region of low interfacial disorder and strong perpendicular magnetic anisotropy, then moving through regions of increasing disorder where the sample undergoes a SRT, and ending (on the right) in the region of large disorder where the magnetization orientation is in plane. As discussed in the previous section, Fig. 2(a) shows that $S(\nu) \sim \nu^{-3 / 2}$ in regions of perpendicular magnetization. As the probe laser is scanned toward the SRT region and perpendicular magnetic anisotropy becomes weaker, $S(\nu)$ continues to exhibit a robust $\nu^{-3 / 2}$ power law. However, the magnitude of $S(\nu)$ and therefore also the integrated noise power, $P=$ $\int S(\nu) d \nu$, increases significantly. Given that the thermal energy $k_{B} T$ is not changing, the increase in thermodynamic fluctuations is consistent with a reduction of the magnetic anisotropy and pinning potential as the SRT is approached.

Crucially, the functional form of the magnetization fluctuation spectrum begins to evolve dramatically upon entering the SRT region. As seen in Fig. 2(a), $S(\nu)$ spectra develop a critical frequency $\nu_{0}$ below which the noise power rolls off and tends toward approximately flat. This critical frequency increases rapidly as the SRT region is traversed. Concomitantly, the integrated noise power $P$ markedly decreases. Beyond the SRT region, where the magnetization is in plane, $\nu_{0}$ exceeds the measurement bandwidth and $S(\nu)$ is small and nearly frequency independent. We emphasize that the spectral shape of $S(\nu)$ and therefore the measured critical frequency $\nu_{0}$ are not affected by the size of the laser spot, indicating that the fluctuating elements are smaller than the spot size. (The magnitude of the measured noise power scales inversely with the probe laser area, as is well known from early studies of spin noise in atomic vapors [36]; however, this is just an overall constant scaling factor-see Supplemental Material [35] 

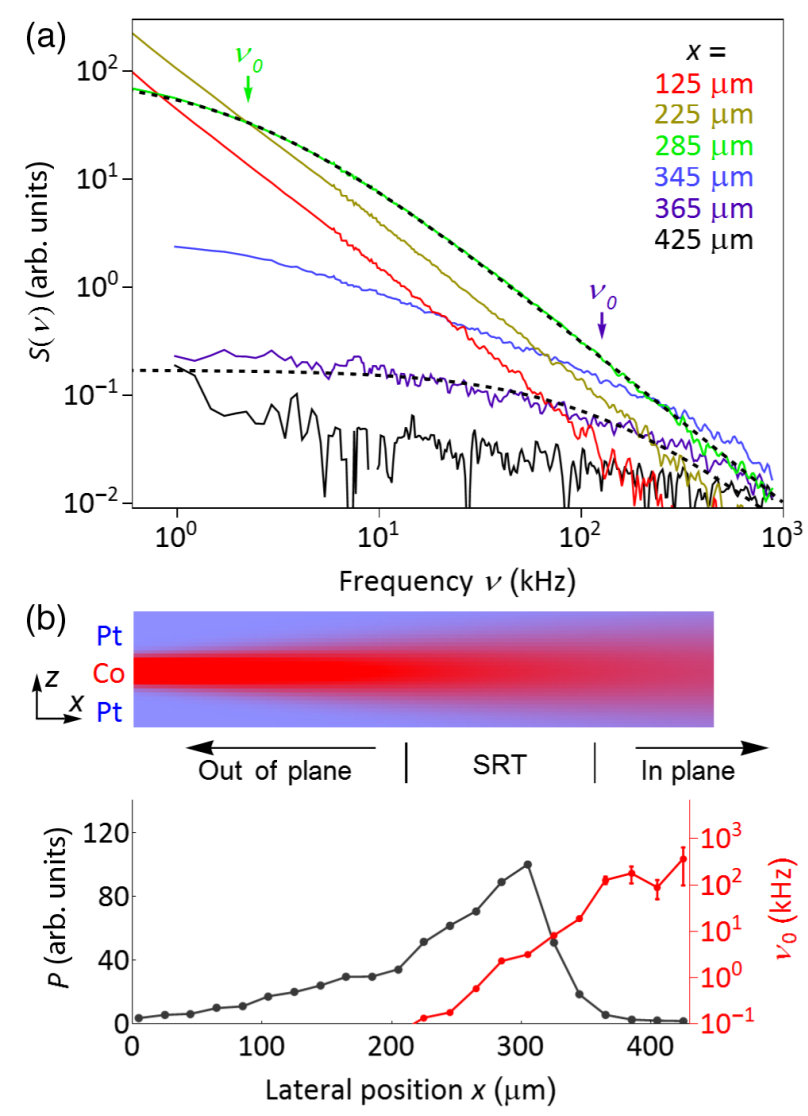

FIG. 2. (a) Power spectra of the magnetization fluctuations $S(\nu)$ at different lateral positions $x$ on the $\mathrm{Pt} / \mathrm{Co} / \mathrm{Pt}$ trilayer. In regions with perpendicular magnetization, $S(\nu) \propto \nu^{-3 / 2}$. Upon entering and traversing the SRT region, $S(\nu)$ develops a steadily increasing critical frequency $\nu_{0}$, below which the noise tends toward spectrally flat, consistent with an evolving low-frequency cutoff in the distribution of fluctuation rates. Empirically, all spectra can be fit by $S(\nu) \propto\left(\nu+\nu_{0}\right)^{-3 / 2}$ (dashed lines). (b) The integrated noise power, $P=\int S(\nu) d \nu$, exhibits a peak in the SRT region. Concomitantly, $\nu_{0}$ rapidly increases through the measurement bandwidth (red points).

for an explicit verification.) Although not the case here, a noise spectrum that changes with spot size could indicate that the fluctuation length scale exceeds the probe size.

Empirically, we find that all of these noise spectra can be reasonably fit over the measured frequency range to the functional form $S(\nu) \propto\left(\nu+\nu_{0}\right)^{-3 / 2}$. Examples of the fits are shown by dashed lines in Fig. 2(a). Although not strictly physical [in principle, $S(\nu)$ should equal $S(-\nu)$ ], this fitting allows us to better identify how the total noise power $P$ and the critical frequency $\nu_{0}$ evolve through the SRT. Figure 2(b) shows how these parameters vary as a function of the lateral position $x$ across the sample. $P$ reaches a maximum at the location where the coercive field $\mu_{0} H_{c} \rightarrow 0$. Near this point, $\nu_{0}$ begins to increase rapidly through the measurement frequency range and $P$ is subsequently rapidly suppressed.

As discussed in the previous section, the presence of $\nu_{0}$ is consistent with the development of a low-frequency truncation, or minimum rate $\gamma_{\min }$, in the distribution $D(\gamma)$ of relaxation rates exhibited by the $\mathrm{Pt} / \mathrm{Co} / \mathrm{Pt}$ film. The slowest out-of-plane magnetization fluctuations $\delta m_{z}(t)$, with characteristic relaxation rate less than $\nu_{0}$ (i.e., those subject to the largest pinning forces), are the first to be suppressed upon entering the SRT region. As the SRT region is traversed and the magnetic anisotropy is further reduced, this low-frequency cutoff increases rapidly through our measurement bandwidth, after which point all out-ofplane fluctuations are very weak and the sample magnetization lies entirely in plane. These trends therefore provide a means of characterizing the loss of perpendicular magnetic order through the spin-reorientation transition by the frequency distribution of the thermodynamic fluctuations.

\section{TEMPERATURE TUNING THROUGH THE SRT}

Because the SRT is defined by the interplay between magnetic anisotropy and thermal energy, we also can expect that the temperature $T$ will have a significant influence on the location of the SRT on the sample [15-17], and therefore on the magnetization fluctuations measured at a fixed location. This is confirmed in Fig. 3, where $S(\nu)$ is measured at a fixed location on the $\mathrm{Pt} / \mathrm{Co} / \mathrm{Pt}$ film as a function of $T$. Effectively, changing $T$ moves the SRT region through the location of the probe laser. We find that $S(\nu)$ first exhibits a $\nu^{-3 / 2}$ power law across the measured $0.1-200 \mathrm{kHz}$ frequency range at low $T$ (where the magnetization is out of plane), then follows a $\left(\nu+\nu_{0}\right)^{-3 / 2}$ dependence at intermediate $T$ (in the SRT regime), and finally evolves at high $T$ towards spectrally flat and small (where the magnetization has switched to in plane).

The effect of increased $T$ on $S(\nu)$ at a fixed location is therefore effectively equivalent to measuring different sample regions with increasing interfacial disorder at fixed $T$ (as in Fig. 2), and again we find that $\nu_{0}$ can be used to characterize the SRT. It is also noteworthy that the exponent of the power-law decay, $\alpha=-3 / 2$, is insensitive to $T$ over the range of the experiment.

At other lateral positions on the sample with different net magnetic anisotropy and pinning potential, the SRT occurs at different temperatures. Qualitatively similar thermodynamic fluctuations are nonetheless obtained (albeit peaked at different temperatures), as shown in Supplemental Material Fig. S1 [35]. In all cases studied, $S(\nu) \propto \nu^{-3 / 2}$ at low $T$, but develop an increasing cutoff frequency $\nu_{0}$ as $T$ is increased through the SRT, highlighting the interplay between the pinning potential and temperature. 

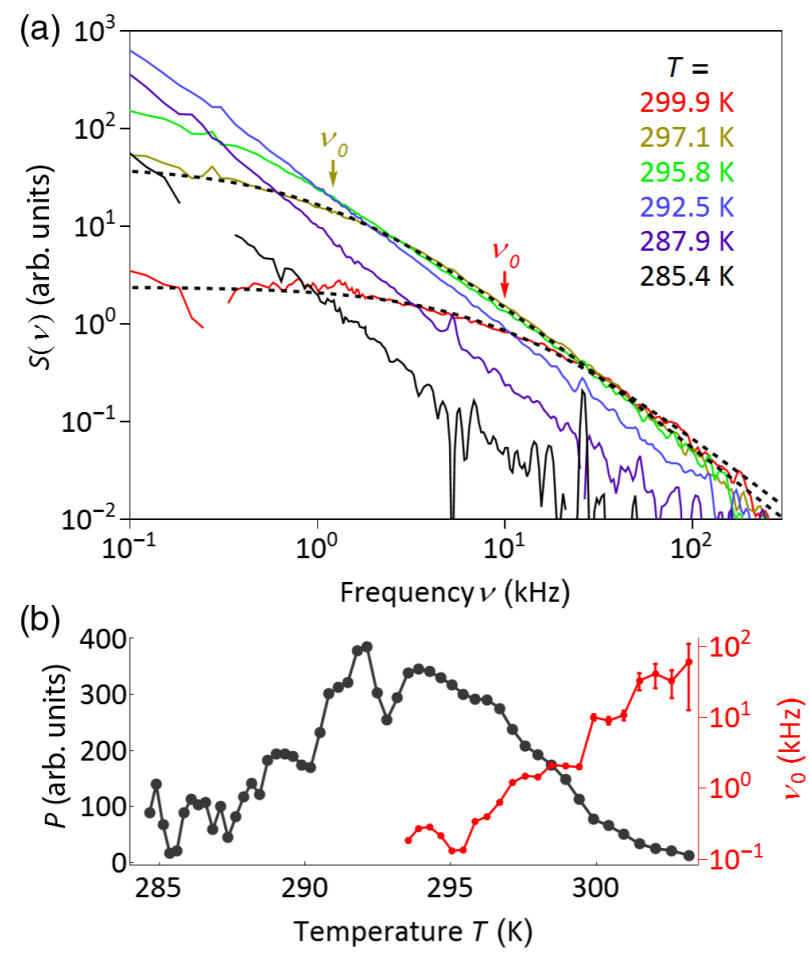

FIG. 3. (a) Magnetization noise spectra $S(\nu)$ at different temperatures $T$, measured at a fixed location on the trilayer that exhibits perpendicular magnetization at low temperatures $(T<290 \mathrm{~K})$, and in-plane magnetization at high temperatures $(T>300 \mathrm{~K}) . S(\nu)$ exhibits robust $\nu^{-3 / 2}$ power-law behavior at low $T$. With increasing $T, S(\nu)$ grows in magnitude but then develops a steadily increasing critical frequency $\nu_{0}$, below which the noise tends toward spectrally flat. Again, all spectra can be empirically fit by $S(\nu) \propto\left(\nu+\nu_{0}\right)^{-3 / 2}$ (dashed lines). (b) Integrated noise power $P$ and critical frequency $\nu_{0}$ versus $T$, indicating that the SRT region has been temperature tuned through the fixed location of the probe.

\section{MAGNETIZATION FLUCTUATIONS IN APPLIED OUT-OF-PLANE FIELDS $B_{z}$, AND RELATION TO THE SUSCEPTIBILITY VIA THE FLUCTUATION-DISSIPATION THEOREM}

Since the magnetization of these $\mathrm{Pt} / \mathrm{Co} / \mathrm{Pt}$ trilayers is strongly field dependent, we next investigate how magnetization fluctuations $\delta m_{z}(t)$ are influenced by applied fields, beginning with the simplest case of purely out-ofplane fields $B_{z}$. Here we expect that sufficiently large $\left|B_{z}\right|$ will completely align and saturate the magnetization $m_{z}$ along $\pm \hat{z}$, thereby suppressing fluctuations. For reference, Fig. 4(a) shows the magnetization $m_{z}\left(B_{z}\right)$ at a location near the SRT, as measured by conventional polar MOKE using a very slow (quasistatic) field sweep rate of $22 \mu \mathrm{T} / \mathrm{s}$. The magnetization exhibits no hysteresis and varies approximately linearly with $B_{z}$ (indicating a constant dc magnetic susceptibility), until it saturates when $\left|B_{z}\right|>B_{\text {sat }}$, where $B_{\text {sat }} \simeq 0.5 \mathrm{mT}$. The absence of hysteresis indicates that at this location the trilayer can relax to an equilibrium (a)
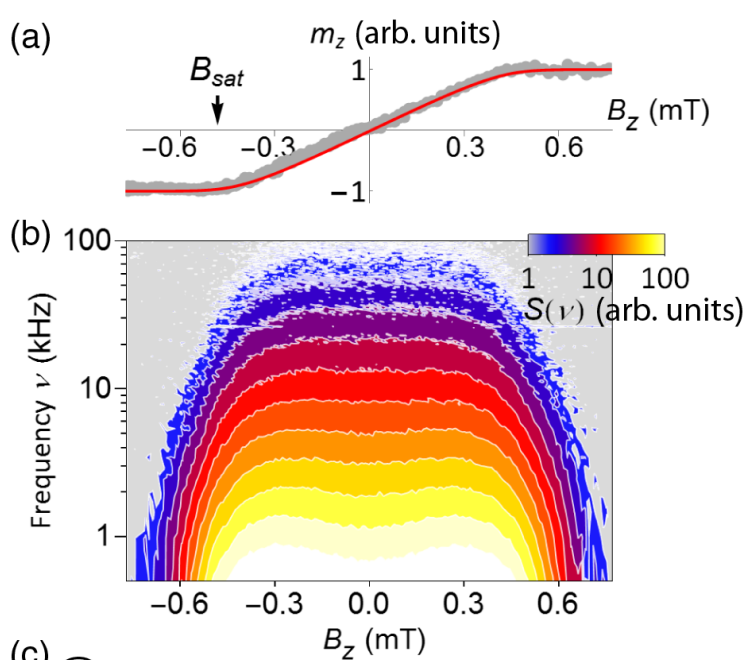

(c)

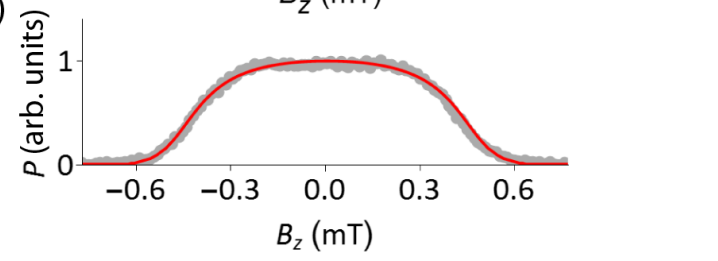

FIG. 4. (a) The average magnetization $m_{z}$ versus applied perpendicular magnetic field $B_{z}$ (measured by conventional MOKE), at a location on the trilayer near the SRT. $m_{z}$ increases linearly with $B_{z}$ (indicating constant magnetic susceptibility $\chi=d m_{z} / d B_{z}$ ), until it saturates for $\left|B_{z}\right|>B_{\text {sat. }}$. (b) Intensity map of the noise spectra $S(\nu)$ at different $B_{z}$. For all $\left|B_{z}\right|<B_{\text {sat }}$, $S(\nu)$ is unaffected (and follows $\nu^{-3 / 2}$ ), but $S(\nu) \rightarrow 0$ when $\left|B_{z}\right|>B_{\text {sat }}$. (c) The integrated magnetization noise $P=$ $\int S(\nu) d \nu$ versus $B_{z}$. The correspondence between $P$ and $\chi$ is in accord with the fluctuation-dissipation theorem. Moreover, the detailed dependence of $P$ (and $m_{z}$ ) on $B_{z}$ can be qualitatively captured by simple considerations of the free energy of a mixture of magnetic domains, as shown by the red lines in (c) and (a); see text.

mazelike magnetic domain pattern on timescales faster than the sweep rate of the external field.

Figure 4(b) shows a color surface map of the fluctuation spectra $S(\nu)$ versus $\nu$ and $B_{z}$, measured at the same location. Note that $S(\nu)$ and $\nu$ are both shown on logarithmic scales, so that equally spaced contours indicate power-law decays. For all small applied fields $\left|B_{z}\right|<B_{\text {sat }}$, $S(\nu)$ spectra are largely unaffected, and they decay with the same power-law exponent $\alpha \simeq-3 / 2$. However, $S(\nu) \rightarrow 0$ at all frequencies when $\left|B_{z}\right|>B_{\text {sat }}$ and the magnetization saturates.

Figure 4(c) shows the integrated magnetization noise power, $P=\int S(\nu) d \nu$, as a function of $B_{z}$. Crucially, we note the close correspondence between $P$ [which is measured via magnetization fluctuation spectroscopy in Fig. 4(b)] and the quasi-dc magnetic susceptibility, $\chi=$ $d m_{z} / d B_{z}$ [which is measured via conventional magnetometry in Fig. 4(a)]. $P$ is large and approximately constant between $\pm B_{\text {sat }}$, where $\chi$ is also large and approximately 
constant. However, both $P$ and $\chi$ are rapidly suppressed to zero when $\left|B_{z}\right|>B_{\text {sat }}$. This correspondence is in line with the fluctuation-dissipation theorem [8], which relates frequency-dependent magnetization fluctuations $S(\nu)$ to the frequency-dependent imaginary (i.e., dissipative) part of the magnetic susceptibility $\chi^{\prime \prime}(\nu)$ :

$$
\frac{2 k_{B} T}{\pi \mu_{0}} \chi^{\prime \prime}(\nu)=\nu S(\nu)
$$

To relate $\chi^{\prime \prime}(\nu)$ to the real dc magnetic susceptibility that was measured in Fig. 4(a), we note that the real and imaginary parts of the susceptibility are necessarily coupled via Kramers-Kronig relations. The dc $(\nu \simeq 0)$ susceptibility is therefore given by

$$
\chi(\nu \simeq 0)=\frac{2}{\pi} \int_{0}^{\infty} \frac{\chi^{\prime \prime}\left(\nu^{\prime}\right)}{\nu^{\prime}} d \nu^{\prime} .
$$

Combining Eqs. (2) and (3), we find that $\chi=d m_{z} /$ $d B_{z} \propto \int S(\nu) d \nu=P$. Significant magnetization noise is therefore expected when the dc magnetic susceptibility is large $[2,30,34]$, in agreement with our measurements.

\section{RELATING THE MAGNETIZATION NOISE TO A SIMPLE MODEL OF THE MAGNETIC FREE ENERGY}

We further note that the field dependence of both the average magnetization $m_{z}$ and the integrated magnetization noise $P$ in Fig. 4 can also be qualitatively and intuitively captured by considering the free energy of a mixture of magnetic domains that are oriented along $\pm \hat{z}$ (i.e., within a domain the average magnetization $m_{z}= \pm 1$ ). We use a simple minimal model to describe the dependence of the free energy, $F=U-T S$, on the average magnetization $m_{z}$. It contains only a quadratic exchange term, a linear Zeeman energy term, and a typical Bragg-Williams mixing term for the entropy $S$ :

$$
\begin{aligned}
F= & a m_{z}^{2}-b B_{z} m_{z}-\frac{1}{2} k_{B} T\left[2 \ln 2-\left(1+m_{z}\right) \ln \left(1+m_{z}\right)\right. \\
& \left.-\left(1-m_{z}\right) \ln \left(1-m_{z}\right)\right],
\end{aligned}
$$

where $a$ and $b$ are scaling constants. We emphasize that Eq. (4) is not intended to represent a microscopic model of magnetization, but rather is intended to simply capture how free energy depends on the macroscopic (area-averaged) $m_{z}$ for the case of Fig. 4, where the film contains a mixture of magnetic domains and exhibits linear susceptibility. Since the data in Fig. 4(a) show no hysteresis and indicate that $m_{z}=0$ when $B_{z}=0, F$ has a minimum here, and therefore we take the coefficient $a$ to be positive. $F\left(m_{z}\right)$ therefore has a single global minimum for any $B_{z}$. At any $B_{z}$, the equilibrium average magnetization $\left\langle m_{z}\right\rangle$ is given approximately by the value of $m_{z}$ for which $F$ is minimized (see
Supplemental Material Fig. S2 [35]). More importantly, the curvature of $F\left(m_{z}\right)$ at this minimum value dictates the magnitude of fluctuations: if $F\left(m_{z}\right)$ increases only slightly for a given fluctuation $\left\langle m_{z}\right\rangle \pm \delta m_{z}$ (i.e., if the curvature $d^{2} F / d m_{z}^{2}$ is small), then fluctuations cost little energy and are thermodynamically likely. In other words, the curvature of $F$ is inversely related to the variance of magnetization fluctuations, $\left(d^{2} F / d m_{z}^{2}\right)^{-1} \sim\left\langle\left(\delta m_{z}\right)^{2}\right\rangle \sim P$ (for details, see Supplemental Material Fig. S2 [35]).

The red lines in Figs. 4(a) and 4(c) show $m_{z}\left(B_{z}\right)$ and $P\left(B_{z}\right)$ calculated using Eq. (4), using a common set of coefficients $a$ and $b$ that are adjusted to match the experimental data. The minimum of $F\left(m_{z}\right)$ occurs at a value of $m_{z}$ that increases approximately linearly with $B_{z}$ until $m_{z}$ approaches \pm 1 , and the curvature of $F\left(m_{z}\right)$ at this minimum is small and relatively constant until it increases sharply as $m_{z} \rightarrow \pm 1$. The overall trends are qualitatively captured, and again establish the link between the magnetization noise power $P$ and the dc magnetic susceptibility $\chi$.

\section{MAPPING MAGNETIZATION FLUCTUATIONS IN BOTH OUT-OF-PLANE AND IN-PLANE MAGNETIC FIELDS}

We also investigate how magnetization fluctuations in $\mathrm{Pt} / \mathrm{Co} / \mathrm{Pt}$ evolve under the additional influence of applied in-plane magnetic fields $B_{x}$. In contrast to out-of-plane fields $B_{z}$, small in-plane fields are not expected to directly contribute to the Zeeman energy of perpendicularly oriented magnetic domains, since $B_{x} \perp \hat{z}$. However, in-plane fields can influence the energy of the domain walls that bound the domains $[49,50]$. The change in domain wall energy at a particular location depends on the relative orientation of $B_{x}$ with respect to the local magnetization direction in the wall. The local energy can therefore increase or decrease. However, when integrating the total domain wall energy around a closed path, linear changes in energy cancel out by symmetry and the total domain wall energy typically decreases quadratically as $B_{x}^{2}[49,50]$. Besides influencing the energy (and fluctuations) of existing domain walls, application of $B_{x}$ at a point very near magnetic saturation can therefore also increase the likelihood of spontaneously forming new magnetic domains (such as bubble domains) [51].

Figures 5(a)-5(d) show intensity plots of the integrated magnetization noise power $P$ as a function of both $B_{z}$ (vertical axis) and also $B_{x}$ (horizontal axis), at four locations on the sample that are separated by only a few microns. Rather striking "bow-tie" structures are revealed (note, however, the factor-of-10 larger field scale for $B_{x}$ ). A vertical line cut through the bow tie at $B_{x}=0$ gives a cross section that corresponds to the plot of $P\left(B_{z}\right)$ shown in Fig. 4(c). The magnetic field at which $P$ falls abruptly to zero is therefore revealed by the upper and lower borders of the bow tie-this is the applied field at which the average 

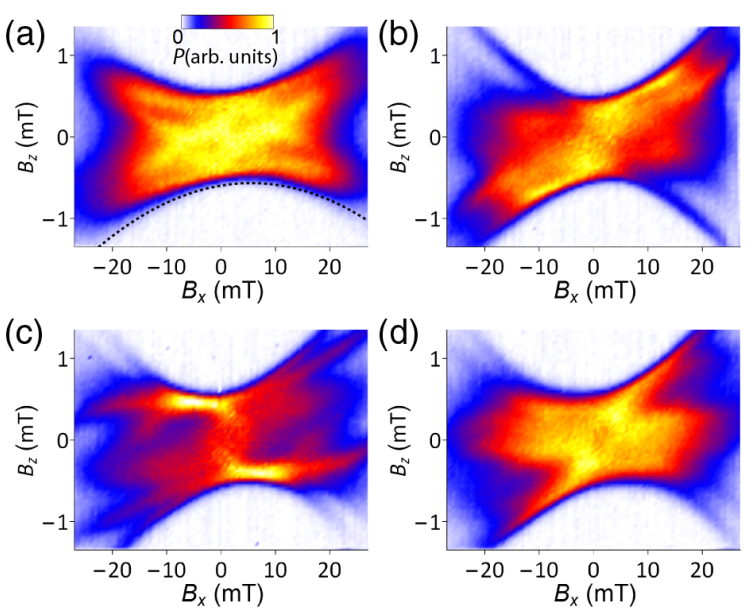

FIG. 5. (a)-(d) Intensity maps of the integrated magnetization noise $P=\int S(\nu) d \nu$ versus in-plane and out-of-plane applied field $\left(B_{x}, B_{z}\right)$, at room temperature $(295 \mathrm{~K})$. The four panels are acquired at slightly different locations on the $\mathrm{Pt} / \mathrm{Co} / \mathrm{Pt}$ film, separated by $<10 \mu \mathrm{m}$. The bow-tie shape of these images is determined by the balance between the fixed thermal energy $k_{B} T$ and the field-dependent energy required to spontaneously form magnetic domains (see text). The very different patterns within each bow tie, and the lack of reflection symmetry about the $B_{x, z}=0$ axes, reveal a spatially varying magnetic anisotropy that is canted slightly away from perfectly out of plane. Dashed line in (a) shows a quadratic fit to the edge of the bow tie.

magnetization $m_{z}$ saturates along $\pm \hat{z}$, which was defined in Fig. 4 as $B_{z}=B_{\text {sat }}$ for the case when $B_{x}=0$.

Several interesting properties of these fluctuation maps are immediately apparent. The first obvious aspect is that magnetization fluctuations persist out to larger $\left|B_{z}\right|$ when $\pm B_{x}$ is applied, and that this relationship is approximately quadratic in $B_{x}$, leading to the characteristic bow-tie shape. This is consistent with the preceding discussion of how the net energy cost to form a closed domain wall decreases as $B_{x}^{2}$ : Consider, for example, a Pt/Co/Pt trilayer in an applied perpendicular field $B_{z}$ slightly larger than $B_{\text {sat }}$, so that $m_{z}$ is saturated, the susceptibility $\chi=d m_{z} / d B_{z}$ is zero, and no magnetic domains are present. The available thermal energy $k_{B} T$ is not quite sufficient to induce the spontaneous formation of oppositely oriented domains, and no fluctuations $\delta m_{z}(t)$ are present. However, when $B_{x}$ is applied, the domain-formation energy falls below $k_{B} T$, at which point domains can spontaneously form and disappear, $m_{z}$ will no longer be saturated, $\chi$ will no longer equal zero, and magnetization fluctuations will appear. Only by increasing $B_{z}$ further (by an amount proportional to $B_{x}^{2}$ ) will the magnetization again achieve saturation.

A second obvious property of these images is that the total noise power $P$ is not constant within a bow tie. Patterns exist, but interestingly the patterns clearly lack reflection symmetry about the horizontal $\left(B_{z}=0\right)$ and vertical axes $\left(B_{x}=0\right)$. Rather, the images exhibit point inversion symmetry about the $B_{x, z}=0$ origin. This is consistent with a uniaxial magnetic anisotropy at the measurement location that is canted slightly away from perfectly out of plane (i.e., it has some in-plane component), so that for a given $B_{z}$ the influence of $+B_{x}$ will in general be different than that of $-B_{x}$ (and similarly, at a given $B_{x}$ the influence of $+B_{z}$ and $-B_{z}$ will be different). However, reversing both $B_{x}$ and $B_{z}$ preserves the symmetry and gives the same magnetic free energy, susceptibility, and noise, in keeping with the images in Fig. 5. Although anisotropic Dzyaloshinskii-Moriya interactions (DMI) are known to exist in perpendicularly oriented ultrathin films $[49,50]$, we believe it is unlikely that DMI generates the anisotropies observed in the noise maps, because on average there are many irregularly shaped domain walls residing within the probe laser spot, with local magnetization orientations sampling all directions in the $x-y$ plane, which should average out. Further, any influence of DMI on these noise maps is expected to be antisymmetric when $B_{x}$ or $B_{z}$ is reversed, which is not observed.

The final aspect that is quite apparent in Fig. 5 is that the four maps of $P\left(B_{x}, B_{z}\right)$ exhibit markedly different patterns, even though they were acquired at only slightly different locations on the $\mathrm{Pt} / \mathrm{Co} / \mathrm{Pt}$ trilayer $(<10 \mu \mathrm{m}$ apart). This indicates that deviations of the magnetic anisotropy away from $\pm \hat{z}$ are strongly position dependent in the $\mathrm{Pt} / \mathrm{Co} / \mathrm{Pt}$ film, at least down to length scales on the order of the laser spot $(4 \mu \mathrm{m})$. We emphasize that in order to acquire a map of $P\left(B_{x}, B_{z}\right)$, the sample is forced to undergo many saturation and demagnetization cycles; therefore, the patterns cannot be due to some temporary or metastable domain configuration but must instead originate in a physical property of the sample. These variations in the magnetic anisotropy landscape likely derive from spatial inhomogeneity in the disorder that is induced by the $\mathrm{Ar}^{+}$irradiation and the fact that $\mathrm{Co}$ and $\mathrm{Pt}$ are not lattice matched.

\section{INDUCING MAGNETIZATION FLUCTUATIONS IN OTHERWISE STABLE FERROMAGNETIC FILMS}

We now investigate how these noise maps evolve over a broad range of magnetic anisotropy. Figure 6 shows maps of $P\left(B_{x}, B_{z}\right)$ acquired at very different lateral positions on the sample, starting in Fig. 6(a) in the region with in-plane magnetization where fluctuations $\delta m_{z}(t)$ are small, continuing in Fig. 6(b) through the SRT region that was discussed in the previous section, and finally moving in Figs. 6(c)-6(e) to less disordered regions of the sample with perpendicular magnetization and increasingly stable out-ofplane ferromagnetism. For reference, the corresponding $m_{z}\left(B_{z}\right)$ magnetization curves (at $B_{x}=0$ ) are also shown. Of particular interest are Figs. 6(c)-6(e), which show how magnetization fluctuations evolve as the trilayer exhibits increasingly strong and stable perpendicular ferromagnetism. The bow ties split apart, with fluctuations appearing at larger $\left|B_{x}\right|$. As expected, no fluctuations are observed near 


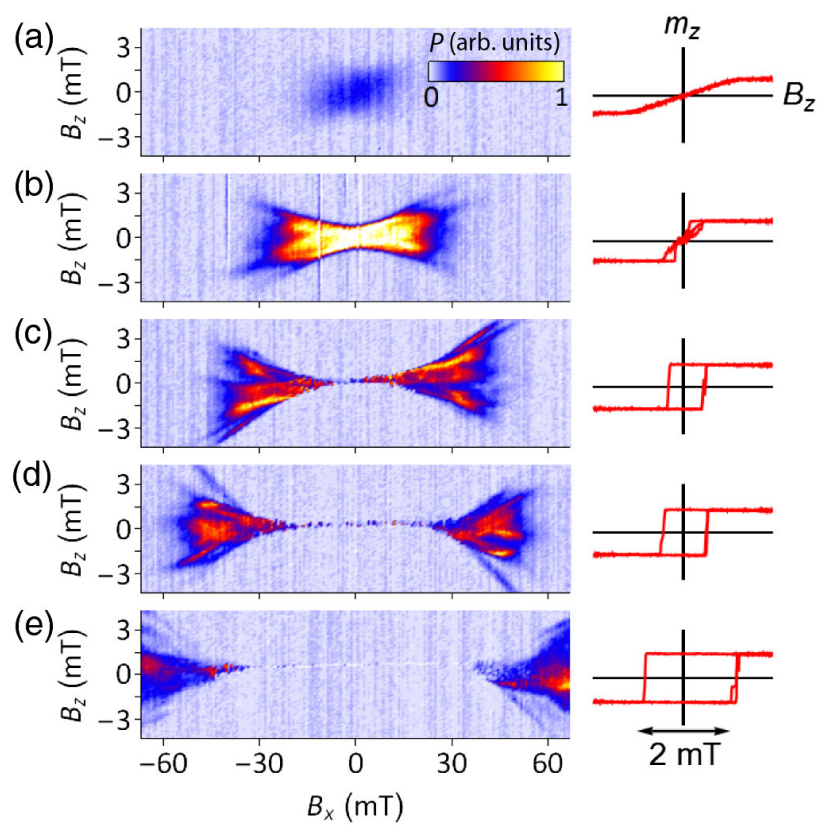

FIG. 6. Intensity maps of the integrated magnetization noise $P\left(B_{x}, B_{z}\right)$ at different locations across the $\mathrm{Pt} / \mathrm{Co} / \mathrm{Pt}$ sample, beginning in (a) regions of the sample with in-plane magnetization, where $P$ is small. (b) Near the SRT, $P$ is large when $\left|B_{z}\right|<$ $B_{\text {sat }}$ (cf. Fig. 5). (c)-(e) In regions with less disorder and therefore increasingly stable perpendicular magnetization, $\mu_{0} H_{c}$ increases and fluctuations vanish at $B_{x, z}=0$. However, strong fluctuations reemerge when larger $\left|B_{x}\right|$ is applied. Note that the $m_{z}\left(B_{z}\right)$ magnetization curves are all acquired at $B_{x}=0$ using a rapidly varying $B_{z}$ (several $\mathrm{Hz}$ ). This gives an open hysteresis loop with large switching (coercive) fields in panels (c)-(e). In the corresponding noise maps, $B_{z}$ was ramped much more slowly $(\mathrm{mHz})$, so that the film switched at very small $B_{z}$.

zero field when the trilayer exhibits stable perpendicular ferromagnetism, which we define as the presence of an open hysteresis loop and a stable remnant magnetization. The key point, however, is that even in trilayers that are nominally very stable ferromagnets at zero field, magnetization fluctuations $\delta m_{z}(t)$ can emerge in sufficiently large in-plane fields $B_{x}$. Studies of nonirradiated trilayers with even larger $\mu_{0} H_{c}$ confirm this to be generally true (see Supplemental Material Fig. S3 [35]).

This result has a number of consequences. Similar to the data shown in Fig. 5, these noise maps show significant nonuniformity, indicating that even films with robust perpendicular ferromagnetism exhibit some degree of microstructured in-plane anisotropy, which could play a role, for example, during magnetic switching in technological applications. Further, it shows that measurements which use fluctuations to detect subtle micromagnetic effects [52] may be possible in materials with significant perpendicular anisotropy. Finally, we speculate that these results may be applicable to future neuromorphic magnetic computation schemes [53], which require the capability to reversibly tune the fluctuations of individual magnetic elements and the interactions between them.

\section{HIGHER-ORDER CORRELATIONS OF THE MAGNETIZATION NOISE}

In this final section, we demonstrate an important consequence and potential advantage of measuring fluctuations $\delta m_{z}(t)$ directly in the time domain, which is that all possible time correlators can, in principle, be retrieved and analyzed from the noise signal. Thus far, we have focused on $S(\nu)$, the power spectral density of the measured fluctuations, which is derived from the second-order (or two-point) time correlator $C_{2}(t)=\left\langle\delta m_{z}(0) \delta m_{z}(t)\right\rangle$. As previously described, $S(\nu)=\left\langle a(\nu) a^{*}(\nu)\right\rangle$, where $a(\nu)=$ $(1 / \sqrt{\tau}) \int_{0}^{\tau} \delta m_{z}(t) e^{i \nu t} d t$ is the Fourier transform of $\delta m_{z}(t)$ measured over a sufficiently long duration $\tau$.

Importantly, however, the $n$ th-order time correlator $C_{n}\left(t_{1}, t_{2}, \ldots, t_{n-1}\right)=\left\langle\delta m_{z}(0) \delta m_{z}\left(t_{1}\right) \ldots \delta m_{z}\left(t_{n-1}\right)\right\rangle$ can contain additional information [54,55] that is not trivially related to $S(\nu)$, particularly in the presence of interactions, inhomogeneous broadening effects, and/or non-Gaussian noise due to (for example) the discrete nature of the system. In general, only the full set of all correlators contains complete information about an interacting system. Higherorder correlations have been studied theoretically $[54,56]$ and experimentally in magnetic systems such as spin glasses [5] and amorphous magnets [57], but we are not aware of any prior experimental studies of higher-order correlations of fluctuations in magnetic films in thermal equilibrium. Such correlators, however, play an important role in the theory of phase transitions and nonlinear thermodynamics [58]. Therefore, their experimental measurement is highly desirable as a tool for characterization of material phases and for tests of fundamental theoretical predictions, such as higher-order fluctuation relations and for testing the universality of scaling exponents.

By way of example, we compute from our noise measurements the simplest nontrivial higher- (third-)order correlator $C_{3}\left(t_{1}, t_{2}\right)$, which can be expressed in the frequency domain as

$$
C_{3}\left(\nu_{1}, \nu_{2}\right)=\left\langle a\left(\nu_{1}\right) a\left(\nu_{2}\right) a^{*}\left(\nu_{1}+\nu_{2}\right)\right\rangle .
$$

$C_{3}$ contains essentially different information compared to a two-point correlator. By construction, it describes correlations between different frequencies and is therefore typically represented as a two-dimensional intensity plot. Furthermore, note that $C_{3}$ is complex valued. Its real and imaginary components have different physical meaning, and in principle, it is possible for fluctuations $\delta m_{z}(t)$ to exhibit a real $C_{3}$, or an imaginary $C_{3}$, or both. The real part of $C_{3}$ is, for example, sensitive to any skewness or third moment in the distribution of $\delta m_{z}(t)$ about its mean-e.g., if a simple magnetic fluctuator spends more time in its $+\hat{z}$ state than in its $-\hat{z}$ state (that being just one example of a 
non-Gaussian noise distribution, as often exemplified by random telegraph noise with statistically inequivalent dwell times in the high and low state).

The imaginary part of $C_{3}$ is sensitive to time-reversal symmetry breaking of the measured signal, meaning that $\delta m_{z}(t) \neq \delta m_{z}(-t)$ in a statistical sense. For example, a sawtooth wave, though not a noise signal per se, breaks time-reversal symmetry, but a triangular wave or sine wave does not. Random telegraph noise with statistically inequivalent rise and fall times would also generate an imaginary $C_{3}$, even when the dwell times in the high and low states are equivalent and the distribution of $\delta m_{z}(t)$ has no skewness. In general, magnetic systems can of course break timereversal symmetry. However, $\operatorname{Im}\left[C_{3}\right]=0$ is expected to hold for Ising-Glauber spin dynamics that satisfy detailed balance at thermal equilibrium [54].

Figure 7 shows the real part of $C_{3}$, measured at a location on the trilayer where $m_{z}\left(B_{z}\right)$ increases linearly [and the noise
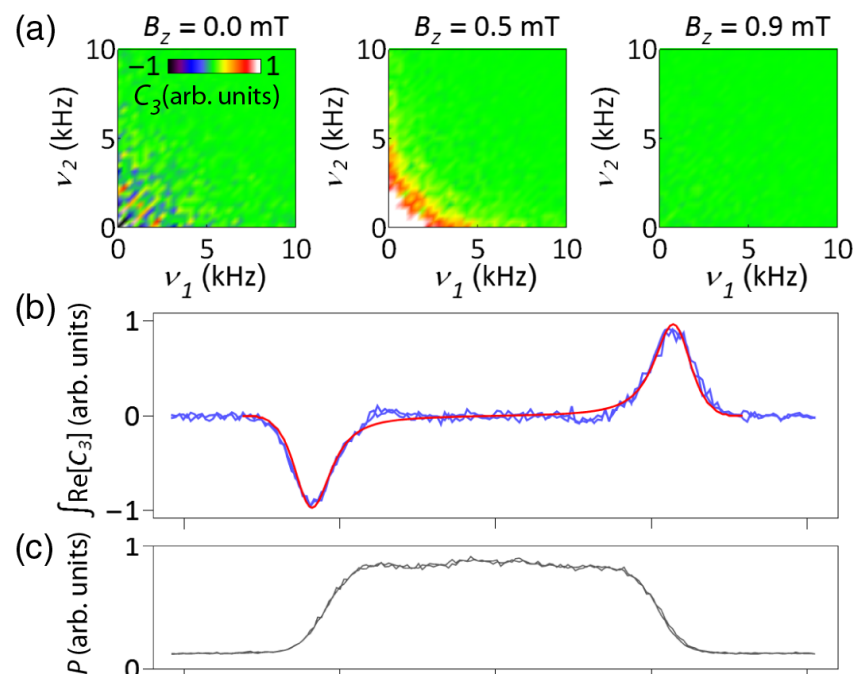

(d)

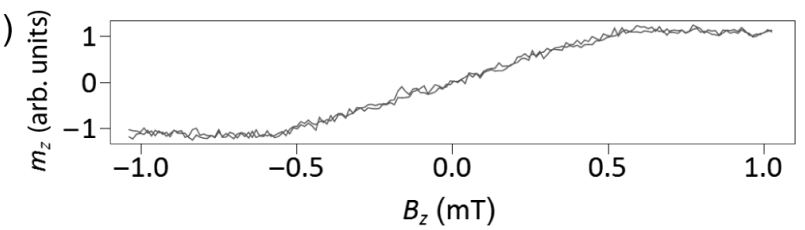

FIG. 7. Measuring higher-order correlations of the magnetization noise at a location on the sample near the SRT that exhibits linear magnetization and large fluctuations (similar to Fig. 4). (a) The real part of the third-order correlator, $\operatorname{Re}\left[C_{3}\left(\nu_{1}, \nu_{2}\right)\right]$, acquired at $B_{z}=0,0.5$, and $0.9 \mathrm{mT}$. (b) The integral $\int \operatorname{Re}\left[C_{3}\right] d \nu_{1} d \nu_{2}$ measured as a continuous function of $B_{z}$. The red line shows this dependence calculated from the magnetic free energy (see Supplemental Material [35] for details). (c) The simultaneously measured total noise power $P=\int S(\nu) d \nu$ versus $B_{z}$ [similar to Fig. 4(c)]. (d) The average magnetization $m_{z}\left(B_{z}\right)$ at this same location, as measured by conventional MOKE, showing a constant susceptibility for $\left|B_{z}\right|<B_{\text {sat }}$ [similar to Fig. 4(a)]. The third-order correlator is approximately zero except when $\left|B_{z}\right| \simeq B_{\text {sat }}$, indicating a skewness in the distribution of $\delta m_{z}(t)$. power $S(\nu)$ is large] when $\left|B_{z}\right|<B_{\text {sat }}$. Fluctuations at this location are very similar to those shown earlier in Fig. 4, where $B_{\text {sat }} \approx 0.5 \mathrm{mT}$. Figure $7\left(\right.$ a) shows $\operatorname{Re}\left[C_{3}\left(\nu_{1}, \nu_{2}\right)\right]$ measured at $B_{z}=0,0.5$, and $0.9 \mathrm{mT}$. Furthermore, Fig. 7(b) shows the integral $\int \operatorname{Re}\left[C_{3}\right] d \nu_{1} d \nu_{2}$ as a continuous function of $B_{z}$. For comparison and completeness, Figs. 7(c) and 7(d) show the simultaneously measured total noise power $P=\int S(\nu) d \nu$ and the average magnetization $m_{z}$ as continuous functions of $B_{z}$ (cf. Fig. 4).

The real part of $C_{3}$ is found to be nonzero when $B_{z} \approx B_{\text {sat }}$ and $m_{z}$ approaches saturation. This indicates a measurable skewness (third moment) in the probability distribution of the noise signal $\delta m_{z}(t)$ about its mean, which can be viewed as arising from a cubic nonlinearity (i.e., a nonzero third derivative) of the free energy $F\left(m_{z}\right)$ at its minimum value. To show this, we note that integrating $\operatorname{Re}\left[C_{3}\left(\nu_{1}, \nu_{2}\right)\right]$ over both frequencies yields the equal-time correlator $\left\langle\delta m_{z}(t)^{3}\right\rangle$, which in turn is proportional to $d^{3} F / d m_{z}^{3}$ (see Supplemental Material Fig. S2 [35]). By inspection of Eq. (4), this cubic nonlinearity arises from the entropic contribution to $F$ when $B_{z} \neq 0$. The data are in good qualitative agreement with predictions from Eq. (4), where $\operatorname{Re}\left[C_{3}\right]$ is small when $\left|B_{z}\right| \ll B_{\text {sat }}$ (because the fluctuations are nearly Gaussian) and when $\left|B_{z}\right| \gg B_{\text {sat }}$ (because the fluctuations are strongly suppressed), but reaches a local maximum when $\left|B_{z}\right| \approx B_{\text {sat }}$, where both the cubic nonlinearity and the fluctuations are large. The red line in Fig. 7(b) shows the real part of $C_{3}$ calculated from the magnetic free energy (derived in detail in the Supplemental Material [35]), showing very good agreement. We further note that other experimental Gaussian noise sources such as photon shot noise do not produce a background level to $C_{3}$ that must be subtracted off [in contrast to $S(\nu)$ ], which further motivates analysis of $C_{3}$ to reveal subtle nonGaussian effects in fluctuation signals.

Finally, we find that $\operatorname{Im}\left[C_{3}\left(\nu_{1}, \nu_{2}\right)\right]=0$ to within our experimental accuracy, for all applied $B_{z}$. This is consistent with—but does not strictly prove-that the measured fluctuation signals $\delta m_{z}(t)$ in our $\mathrm{Pt} / \mathrm{Co} / \mathrm{Pt}$ trilayers obey time-reversal symmetry, and that in thermal equilibrium $\delta m_{z}(t)$ remained indistinguishable from $\delta m_{z}(-t)$ in a statistical sense.

\section{SUMMARY}

We used Kerr magnetometry to study the broadband frequency spectra of thermodynamic magnetization fluctuations in an archetypal ultrathin ferromagnet $(\mathrm{a} \mathrm{Pt} / \mathrm{Co} / \mathrm{Pt}$ trilayer). The power spectral density of the fluctuations $S(\nu)$ is found to follow a robust $\nu^{-3 / 2}$ power law on regions of the trilayer exhibiting out-of-plane magnetization, indicating a broad distribution of fluctuation and relaxation rates. However, the functional form of $S(\nu)$ changes dramatically as the magnetic anisotropy and therefore pinning is reduced to zero and the trilayer is tuned through 
a SRT (either by increasing temperature or by increasing interfacial disorder). Namely, as the SRT is traversed, $S(\nu)$ develops a steadily increasing critical frequency $\nu_{0}$ below which the noise power tends towards spectrally flat, indicating an evolving low-frequency cutoff for out-ofplane magnetization fluctuations. These results therefore provide a means of characterizing phase transitions in ferromagnets via their influence on spontaneous dynamics. The fluctuations are found to be strongly dependent on applied magnetic fields, which can be understood within the framework of the fluctuation-dissipation theorem and from considerations of the magnetic free energy. Sufficiently large in-plane fields can induce spontaneous fluctuations in films that otherwise exhibit stable outof-plane ferromagnetism. Finally, we demonstrate that higher-than-second-order correlators can be computed and analyzed from the measured fluctuations, which can potentially provide a sensitive and powerful means of revealing subtle influences of non-Gaussian noise and time-reversal breaking effects.

\section{ACKNOWLEDGMENTS}

We thank Dan Pierce, Vivien Zapf, Mark Stiles, Alice Mizrahi, and Carl Boone for useful discussions. Work at the NHMFL was supported by the Los Alamos LDRD program, National Science Foundation DMR-1157490, the State of Florida, and the U.S. Department of Energy.

[1] H. Bittel, Noise of Ferromagnetic Materials, IEEE Trans. Magn. 5, 359 (1969).

[2] M. Ocio, H. Bouchiat, and P. Monod, Observation of $1 / f$ Magnetic Fluctuations in Spin Glasses, J. Magn. Magn. Mater. 54-57, 11 (1986).

[3] T. J. Silva and H. N. Bertram, Magnetization Fluctuations in Uniformly Magnetized Thin-Film Recording Media, IEEE Trans. Magn. 26, 3129 (1990).

[4] N. Smith and P. Arnett, White-Noise Magnetization Fluctuations in Magnetoresistive Heads, Appl. Phys. Lett. 78, 1448 (2001).

[5] M. B. Weissman, What Is a Spin Glass? A Glimpse via Mesoscopic Noise, Rev. Mod. Phys. 65, 829 (1993).

[6] C. P. Bean and J. D. Livingston, Superparamagnetism, J. Appl. Phys. 30, S120 (1959).

[7] O. Sinwani, J. W. Reiner, and L. Klein, Monitoring Superparamagnetic Langevin Behavior of Individual $\mathrm{SrRuO}_{3}$ Nanostructures, Phys. Rev. B 89, 020404(R) (2014).

[8] R. Kubo, The Fluctuation-Dissipation Theorem, Rep. Prog. Phys. 29, 255 (1966).

[9] M. T. Johnson, P. J. H. Bloemen, F. J. A. den Broeder, and J. J. de Vries, Magnetic Anisotropy in Metallic Multilayers, Rep. Prog. Phys. 59, 1409 (1996).

[10] C. A. F. Vaz, J. A. C. Bland, and G. Lauhoff, Magnetism in Ultrathin Film Structures, Rep. Prog. Phys. 71, 056501 (2008).
[11] R. Allenspach and A. Bischof, Magnetization Direction Switching in $\mathrm{Fe} / \mathrm{Cu}(100)$ Epitaxial Films: Temperature and Thickness Dependence, Phys. Rev. Lett. 69, 3385 (1992).

[12] Z. Q. Qiu, J. Pearson, and S. D. Bader, Asymmetry of the Spin Reorientation Transitition in Ultrathin Fe Films and Wedges Grown on Ag(100), Phys. Rev. Lett. 70, 1006 (1993).

[13] C. Chappert, H. Bernas, J. Ferré, V. Kottler, J.-P. Jamet, Y. Chen, E. Cambril, T. Devolder, F. Rousseaux, V. Mathet, and H. Launois, Planar Patterned Magnetic Media Obtained by Ion Irradiation, Science 280, 1919 (1998).

[14] J. Fassbender, D. Ravelosona, and Y. Samson, Tailoring Magnetism by Light-Ion Irradiation, J. Phys. D 37, R179 (2004).

[15] D. P. Pappas, K. P. Kämper, and H. Hopster, Reversible Transition between Perpendicular and In-Plane Magnetization in Ultrathin Films, Phys. Rev. Lett. 64, 3179 (1990).

[16] D. Pescia and V. L. Pokrovsky, Perpendicular versus InPlane Magnetization in a 2D Heisenberg Monolayer at Finite Temperatures, Phys. Rev. Lett. 65, 2599 (1990).

[17] P. J. Jensen and K. H. Bennemann, Direction of the Magnetization of Thin Films and Sandwiches as a Function of Temperature, Phys. Rev. B 42, 849 (1990).

[18] Y. Millev and J. Kirschner, Reorientation Transitions in Ultrathin Ferromagnetic Films by Thickness- and Temperature-Driven Anisotropy Flows, Phys. Rev. B 54, 4137 (1996).

[19] A. Hubert and R. Schäfer, Magnetic Domains: The Analysis of Magnetic Microstructures (Springer Science \& Business Media, Berlin, 2008).

[20] Y. Yafet and E. M. Gyorgy, Ferromagnetic Strip Domains in an Atomic Monolayer, Phys. Rev. B 38, 9145 (1988).

[21] Y.Z. Wu, C. Won, A. Scholl, A. Doran, H. W. Zhao, X. F. Jin, and Z. Q. Qiu, Magnetic Stripe Domains in Coupled Magnetic Sandwiches, Phys. Rev. Lett. 93, 117205 (2004).

[22] C. Won, Y. Z. Wu, J. Choi, W. Kim, A. Scholl, A. Doran, T. Owens, J. Wu, X.F. Jin, H. W. Zhao, and Z. Q. Qiu, Magnetic Stripe Melting at the Spin Reorientation Transition in FeNiCu(001), Phys. Rev. B 71, 224429 (2005).

[23] M. Kronseder, M. Buchner, H. G. Bauer, and C. H. Back, Dipolar-Energy-Activated Magnetic Domain Pattern Transformation Driven by Thermal Fluctuations, Nat. Commun. 4, 2054 (2013).

[24] D. Venus, C. S. Arnold, and M. Dunlavy, Domains in Perpendicularly Magnetized Ultrathin Films Studied Using the Magnetic Susceptibility, Phys. Rev. B 60, 9607 (1999).

[25] L. Belliard, J. Miltat, V Kottler, V. Mathet, and C. Chappert, Stripe Domains Morphology versus Layers Thickness in CoPt Multilayers, J. Appl. Phys. 81, 5315 (1997).

[26] N. Bergeard, J. P. Jamet, A. Mougin, J. Ferré, J. Gierak, E. Bourhis, and R. Stamps, Dynamic Fluctuations and TwoDimensional Melting at the Spin Reorientation Transition, Phys. Rev. B 86, 094431 (2012).

[27] F. Ando, H. Kakizakai, T. Koyama, K. Yamada, M. Kawaguchi, S. Kim, K.-J. Kim, T. Moriyama, D. Chiba, and T. Ono, Modulation of the Magnetic Domain Size Induced by an Electric Field, Appl. Phys. Lett. 109, 022401 (2016).

[28] M. Yamanouchi, A. Jander, P. Dhagat, S. Ikeda, F. Matsukura, and H. Ohno, Domain Structure in CoFeB Thin Films with 
Perpendicular Magnetic Anisotropy, IEEE Magn. Lett. 2, 3000304 (2011).

[29] O. Portmann, A. Vaterlaus, and D. Pescia, Observation of Stripe Mobility in a Dipolar Frustrated Ferromagnet, Phys. Rev. Lett. 96, 047212 (2006).

[30] T. N. G. Meier, M. Kronseder, M. Zimmermann, and C. H. Back, Quantification of Thermal Fluctuations in Stripe Domain Patterns, Phys. Rev. B 93, 064424 (2016).

[31] M. Kronseder, T. N. G. Meier, M. Zimmermann, M. Buchner, M. Vogel, and C. H. Back, Real-Time Observation of Domain Fluctuations in a Two-Dimensional Magnetic Model System, Nat. Commun. 6, 6832 (2015).

[32] A. L. Balk, M. D. Stiles, and J. Unguris, Critical Behavior of Zero-Field Magnetic Fluctuations in Perpendicularly Magnetized Thin Films, Phys. Rev. B 90, 184404 (2014).

[33] K. A. Seu, S. Roy, J. J. Turner, S. Park, C. M. Falco, and S. D. Kevan, Cone Phase and Magnetization Fluctuations in $\mathrm{Au} / \mathrm{Co} / \mathrm{Au}$ Thin Films Near the Spin-Reorientation Transition, Phys. Rev. B 82, 012404 (2010).

[34] Z. Diao, E. R. Nowak, G. Feng, and J. M. D. Coey, Magnetic Noise in Structured Hard Magnets, Phys. Rev. Lett. 104, 047202 (2010).

[35] See Supplemental Material at http://link.aps.org/ supplemental/10.1103/PhysRevX.8.031078 for temperature-dependent noise measurements at different locations, a description of a free energy model for the second and third moments of the fluctuations, data showing field-induced magnetization fluctuations on samples with higher coercive field, confirmation of the scaling of the noise power with probe laser area, and a discussion of measurement uncertainties.

[36] S. A. Crooker, D. G. Rickel, A. V. Balatsky, and D. L. Smith, Spectroscopy of Spontaneous Spin Noise as a Probe of Spin Dynamics and Magnetic Resonance, Nature (London) 431, 49 (2004).

[37] M. Oestreich, M. Römer, R. J. Haug, and D. Hägele, Spin Noise Spectroscopy in GaAs, Phys. Rev. Lett. 95, 216603 (2005).

[38] S. A. Crooker, J. Brandt, C. Sandfort, A. Greilich, D. R. Yakovlev, D. Reuter, A. D. Wieck, and M. Bayer, Spin Noise of Electrons and Holes in Self-Assembled (In,Ga)As Quantum Dots, Phys. Rev. Lett. 104, 036601 (2010).

[39] V. S. Zapasskii, A. Greilich, S. A. Crooker, Yan Li, G. G. Kozlov, D. R. Yakovlev, D. Reuter, A. D. Wieck, and M. Bayer, Optical Spectroscopy of Spin Noise, Phys. Rev. Lett. 110, 176601 (2013).

[40] W. H. Press, Flicker Noises in Astronomy and Elsewhere, Comments Astrophys. 7, 103 (1978).

[41] M. B. Weissman, 1/f Noise and Other Slow, Nonexponential Kinetics in Condensed Matter, Rev. Mod. Phys. 60, 537 (1988).

[42] E. Milotti, Linear Processes that Produce 1/f or Flicker Noise, Phys. Rev. E 51, 3087 (1995).

[43] B. Alessandro, C. Beatrice, G. Bertotti, and A. Montorsi, Domain-Wall Dynamics and Barkhausen Effect in Metallic
Ferromagnetic Materials. I. Theory, J. Appl. Phys. 68, 2901 (1990).

[44] P. Cizeau, S. Zapperi, G. Durin, and H. E. Stanley, Dynamics of a Ferromagnetic Domain Wall and the Barkhausen Effect, Phys. Rev. Lett. 79, 4669 (1997).

[45] M. C. Kuntz and J. P. Sethna, Noise in Disordered Systems: The Power Spectrum and Dynamic Exponents in Avalanche Models, Phys. Rev. B 62, 11699 (2000).

[46] O. Narayan, Self-Similar Barkhausen Noise in Magnetic Domain Wall Motion, Phys. Rev. Lett. 77, 3855 (1996).

[47] F. Bohn, M. A. Correa, M. Carara, S. Papanikolaou, G. Durin, and R. L. Sommer, Statistical Properties of Barkhausen Noise in Amorphous Ferromagnetic Films, Phys. Rev. E 90, 032821 (2014).

[48] A. Singh, S. Mukhopadhyay, and A. Ghosh, Tracking Random Walk of Individual Domain Walls in Cylindrical Nanomagnets with Resistance Noise, Phys. Rev. Lett. 105, 067206 (2010).

[49] A. Thiaville, S. Rohart, E. Jue, V. Cros, and A. Fert, Dynamics of Dzyaloshinskii Domain Walls in Ultrathin Magnetic Films, Europhys. Lett. 100, 57002 (2012).

[50] S.-G. Je, D.-H. Kim, S.-C. Yoo, B.-C. Min, K.-J. Lee, and S.-B. Choe, Asymmetric Magnetic Domain-Wall Motion by the Dzyaloshinskii-Moriya Interaction, Phys. Rev. B 88, 214401 (2013).

[51] J. Choi, J. Wu, C. Won, Y.Z. Wu, A. Scholl, A. Doran, T. Owens, and Z. Q. Qiu, Magnetic Bubble Domain Phase at the Spin Reorientation Transition of Ultrathin $\mathrm{Fe} / \mathrm{Ni} /$ $\mathrm{Cu}(001)$ Film, Phys. Rev. Lett. 98, 207205 (2007).

[52] V. E. Demidov, S. Urazhdin, E. R. J. Edwards, M. D. Stiles, R. D. McMichael, and S. O. Demokritov, Control of Magnetic Fluctuations by Spin Current, Phys. Rev. Lett. 107, 107204 (2011).

[53] A. Mizrahi, N. Locatelli, R. Matsumoto, A. Fukushima, H. Kubota, S. Yuasa, V. Cros, J.-V. Kim, J. Grollier, and D. Querlioz, Magnetic Stochastic Oscillators: Noise-Induced Synchronization to Underthreshold Excitation and Comprehensive Compact Model, IEEE Trans. Magn. 51, 1 (2015).

[54] F. Li, A. Saxena, D. Smith, and N. A. Sinitsyn, HigherOrder Spin Noise Statistics, New J. Phys. 15, 113038 (2013).

[55] S. Starosielec, R. Fainblat, J. Rudolph, and D. Hägele, TwoDimensional Higher-Order Noise Spectroscopy Up to Radio Frequencies, Rev. Sci. Instrum. 81, 125101 (2010).

[56] P. Schad, B. N. Narozhny, Gerd Schön, and A. Shnirman, Nonequilibrium Spin Noise and Noise of Suceptibility, Phys. Rev. B 90, 205419 (2014).

[57] J. R. Petta, M. B. Weissman, and G. Durin, Barkhausen Pulse Structure in an Amorphous Ferromagnet: Characterization by High-Order Spectra, Phys. Rev. E 57, 6363 (1998).

[58] R. L. Stratonovich, Nonlinear Nonequilibrium Thermodynamics I (Springer-Verlag, Berlin, 1992). 